\title{
New techniques for the non-contact measurement of displacer motion of a miniature split-Stirling cryocooler
}

\author{
Y.P. Yang, B.J. Huang, F.M. Chen, S.B. Chien* and T.F. Shieh* \\ Department of Mechanical Engineering, National Taiwan University, Taipei, Taiwan, \\ R.O.C. \\ *Chung-Shen Institute of Technology, Lung-Tung, Taiwan, R.O.C.
}

Received 4 August 1995; revised 9 October 1995

This paper proposes optional methods to calibrate the static sensitivity of the measurement of displacer motion inside the cold finger of a split-Stirling cryocooler. The relative displacement measurement method, as previously proposed, required that the regenerator matrix material should have such a high magnetic permeability that the linear variable-differential transformer (LVDT) could measure the displacer motion without large signal amplification. This would be impossible for low-permeability materials, e.g. stainless steel $316 \mathrm{~L}$ and $304 \mathrm{~L}$, contained in the displacer. Therefore, three options are proposed for the calibration of static sensitivity: contact, purge or dynamic calibration methods. Temperature compensation is also found to be necessary to transfer the LVDT voltage to the true displacer displacement when performing the non-contact measurement of the displacer motion. (c) 1996 Elsevier Science Limited

Keywords: split-Stirling cryocooler; static sensitivity calibration

Split-type Stirling cryocoolers are characterized by complete separation of the expansion cylinder from the compressor cylinder, drive motor and crankcase. These cryocoolers have been made small, lightweight, of low power and more efficient in terms of coefficient of performance (COP), to provide a cryogenic environment for cold electronics. The fine-bore gas-filled metal tube that connects the expansion and compression cylinders is sufficiently malleable. This not only permits the low-mass component to be mounted in such applications as ultra-scnsitive magnetometers and gigaflop-speed supercomputers, but also results in the isolation of cold electronic systems from environment noise, compressor-induced vibration and electromagnetic emissions from the compressor and the motor. Therefore, the displacer in the expansion cylinder is free and oscillates simply as a result of the gas force acting on it. The gas force is provided by the actuating piston oscillating in the compression cylinder with a phase-shift relative to the displacer motion. The stroke variation of the displacer indicates the volume change in the expansion space, and its pressure-volume diagram illustrates the work done by the gas on the displacer. The net area of the diagram will represent the heat transferred to the expansion space, a positive work, so there is some refrigeration effect, and the tip of the cold finger will become cold.

In the design of a split-type miniature Stirling cryocooler, the displacer motion is closely related to the available refrigeration ${ }^{\prime}$, which depends upon the cooling or ambient atmospheric temperature, working gas pressure, the swept volume in the compression space or the expansion space, the operating frequency, etc. The measurement of the displacer's displacement, therefore, becomes essential during the development of split-type Stirling cryocoolers, from the prototype testing to the quality control process of the product.

Xiang et $a .^{2}$ took the displacer motion signal to identify the movement damping of the displacer, which changes along with the lowering of the refrigeration temperature, in a split-cycle free-piston Stirling cryocooler. Zhang et al. ${ }^{3}$ conducted an experimental investigation on the dynamic pressure distribution in a split-piston Stirling cryocooler system, where the displacer motion was measured with a Hall-effect displacement transducer. Stolfi and de Jonge ${ }^{4}$ regulated the motions of the displacer and the piston of a Briarcliff cryocooler by feeding their displacement signals back into a control system to adjust the current in the linear motors. Capacitive position pickups were also used to measure both the compressor piston and displacer motions in BAe $20-80 \mathrm{~K}$ split-Stirling coolers for the control system to maintain the required phase relationship between the compressor and the displacer ${ }^{5}$. The above measurement methods rely on built-in sensors installed inside the system of a closed chamber.

Previously proposed in this research was the relative displacement measurement method to calibrate the static sensitivity of the non-contact measurement of the displacer 
motion by the use of the well-known linear variable-differential transformer (LVDT) ${ }^{6}$. The displacement of the displacer inside the cold finger was measured with neither built-in sensors nor sensors directly contacting the displacer. However, the relative displacement measurement method calibrating the static sensitivity requires that the regenerator matrix material and the cap of the displacer have such a high magnetic permeability that the LVDT can measure the displacer motion without large signal amplification. This will be impossible for regenerator matrix materials, such as stainless steel $316 \mathrm{~L}$ and $304 \mathrm{~L}$, of low magnetic permeability. Therefore, three options are proposed in this paper for the calibration of static sensitivity of the measurement of motion of the displacer with low magnetic permeability: contact, purge or dynamic calibration methods. Moreover, the magnetic permeability of the stainless steel reaches a maximum as the temperature approaches the Néel temperature, that corresponds to the transition point from the anti-ferromagnetic state to the paramagnetic state and vice versa, around $20 \mathrm{~K}$ or so ${ }^{7}$. The static sensitivity must be compensated for the change in the operating temperature so as to transfer the I.VDT voltage to the true displacer displacement while performing the noncontact measurement of the displacer motion.

\section{Relative displacement measurement method and its difficulties}

Linear variable-differential transformers (LVDTs) are used to measure displacement by the principle of electromagnetic induction, and are termed variable-inductance transducers. The basic arrangement of the LVDT constitutes a primary winding and a secondary winding, symmetrically wound in the two end segments. The primary coil carries an $\mathrm{AC}$ excitation that induces a steady $\mathrm{AC}$ voltage in the two secondary coils connected in series opposition. The level of the induced voltage depends upon the flux linkage between coils. The reluctance of the flux path varies with the position of the iron core due to its relative permeability, which indicates how a magnetic material changes the magnetic induction compared with the induction that would be observed in free space. The iron core is used to connect the object whose position is to be measured, and the voltage induced in the two secondary coils varies with the position of the iron core. This core is usually included in commercially available LVDTs, and is made of ferromagnetic material inserted coaxially into the cylindrical, insulating, non-magnetic form without actually touching it. With builtin microelectronics or some readout systems for signal conditioning. either by the rectification or the demodulation method $^{8}$, the output is directly proportional, in the linear operating range, to the displacement.

As in a previous paper $^{6}$, a different way of using LVDT in measuring the position of the displacer was developed. The reason why, in this research, the authors used the LVDT instead of other sensors, like optical displacement sensors or built-in Hall-effect sensors, are as follows.

1 The beam from the optical sensor cannot penetrate the stainless steel cylinder wall of the cold finger.

2 Built-in sensors increase the cost and complexity in the product design and manufacture, and are redundant when control on the displacer motion is not required.

3 Neither installation of sensors inside the cooler nor destruction of the cylinder wall is required when the LVDT measurement is performed.

The LVDT housing containing the coils was mounted outside the cold-finger cylinder, inside which the displacer now plays the role of the commercial iron core. The cylinder wall is usually made of 300 series stainless steel with low permeability. Displacers were normally equipped with regenerator matrix composed of any finely divided material ${ }^{8,9}$. Difficulties in measuring the displacement occur due to the low permeability and shielding effect of the cold finger, resulting in the attenuation of the induced voltage in the secondary coils of the LVDT. Therefore, the output signal must be calibrated to identify the input signal - the true displacement of the displacer motion. When an inputoutput calibration has been performed, the static sensitivity (or simply sensitivity) $K$ can be defined as the gradient of the calibration curve ${ }^{10}$, i.e.

$K=\frac{\Delta x}{\Delta V}$

as an indication of the change of true displacement $\Delta x$ per unit voltage output $\Delta V$. It is apparent that $K$ is a function of the magnetic permeability of the regenerator matrix, cylinder wall thickness, ambient temperature, operating frequency of the Stirling cryocooler, etc. As a result, the true displacement of the displacer can be expressed as

$x(t)=K\left[V(t)-V_{0}\right]$

where $V(t)$ is the voltage output of the LVDT, and $V_{0}$ is a reference voltage for the zero position of the displacer at an ambient temperature.

The relative displacement measurement method was proposed in a previous paper ${ }^{6}$ to calibrate the measurement sensitivity $K$. However, there are several disadvantages.

1 If the regenerator matrix and the cap of its container have very low magnetic permeability compared with ferromagnetic materials, the signal-to-noise ratio of measurement through the LVDT becomes very small with respect to the background voltage.

2 The background voltage varies along the axial direction of the cold finger, so that the static sensitivity of the LVDT is nol linear. However, better calibration of the relative displacement method requires a larger linear range of measurement in terms of LVDT locations.

3 The background voltage, being non-negligible compared with the induced voltage by the displacer motion, must be measured as the displacer is disassembled. But, this is not practical or may not be possible for nondestructive measurement.

4 The cylinder wall thickness of the cold finger produces a shielding effect on the induced change of the flux linkage in the secondary coils.

5 The relative displacement measurement method requires that the LVDT should move along outside the cold cylinder. The length of the displacer is usually greater than the standard iron core of the LVDT, therefore the displacer motion might fall outside the linear range of the LVDT.

6 The output voltage of the LVDT is very sensitive to the temperature of the tip of the cold finger, since the flux 
permeability of the regenerator matrix tends to increase as the material temperature decreases ${ }^{7}$.

In fact, the standard core of the LVDT is made of iron whose relative permeability is about $4000^{11}$. The regenerator matrix in the displacer that we designed for the splitStirling cryocooler is made of stainless steel $316 \mathrm{~L}$, whose relative permeability is 1.005 as an average low-frequency value at room temperature ${ }^{7}$. Since the displacer now plays the role of a commercial iron core, the sensitivity of the non-contact measurement of the displacer motion must be reduced at least to $1 / 4000$, which is $32 / 4000 \mathrm{mV} \mathrm{mm}^{-1}$ according to the specification of the LVDT listed in the Appendix. It is still possible to measure the displacer motion with a proper signal amplifier. But the background voltage of the cold cylinder, which is also made of stainless steel $316 \mathrm{~L}$, becomes important and varies nonlinearly along the cold cylinder. Therefore, more modification and effort must be made to calibrate the measurement sensitivity using the relative displacement measurement method, and there is no guarantee of the accuracy of such a complicated measurement with more signal manipulation.

In this research, the authors propose three optional methods for the calibration of sensitivity to overcome difficulties in the measurement method and limitations of sensors. The accuracy and reliability of each method are also discussed.

\section{Sensitivity calibration methods}

\section{Contact calibration}

The purpose of the contact calibration of sensitivity is to verify that, at any LVDT location, the calibrated sensitivity will be the same as that calibrated by the purge and dynamic methods. The latter methods will be elaborated.

The experimental setup, as shown in Figure 1, consists of a split-Stirling cryocooler, LVDT, demodulator, signal analyser and temperature indicator. As the LVDT surrounds the cold finger at a certain position, the bottom cap of the cold finger is removed and the displacer can be shifted inside the cylinder. The displacement of the displacer is measured by a caliper vernier with resolution of $0.002 \mathrm{~mm}$, and the corresponding voltage output is recorded. Different locations of LVDT are measured by a micrometer of resolution $0.0002 \mathrm{~mm}$.

Figure 2 shows that the relationship between the output voltage and position of the displacer is not truly linear. For linear curve fitting, the linear correlation coefficient presents the degree of linearity. As shown in Table 1 , the static sensitivity of measurement changes for various LVDT positions from the base end of the cold finger as shown in Figure 3. We found that the maximum calibration error occurred when the displacer was close to the cold tip, at which the sensitivity reduced. This is reasonable because the cold-cap material, copper alloy, shields from more magnetic flux than the cylinder wall, so that the induced voltage in the secondary coils of the LVDT becomes smaller.

\section{Purge calibration}

It is not convenient to remove the bottom cap of the cold finger for the contact calibration, neither is it possible to do so during the quality control of products. What we desire is to calibrate the Ineasurement sensitivity in the manufac- turing process. We found that, during the working gas helium $(\mathrm{He})$ charge and purge process of the Stirling cryocooler, the displacer moved to the upper and lower dead points in the cold cylinder under the action of instantaneous pressure differences. Since the stroke between the upper and lower dead points is known as a design specification $(5.31 \mathrm{~mm})$, the measurement sensitivity can be obtained by dividing the stroke by the voltage difference between those two dead points. For the LVDT position at $28 \mathrm{~mm}$ from the base of the cold finger, under the ambient temperature of $18^{\circ} \mathrm{C}$, the average sensitivity for 16 independent measurements is $11.9977 \pm 0.1479 \mathrm{~mm} \mathrm{~V}^{-1}$ with $95 \%$ confidence. It is worth noting that the sensitivity of $12.12 \mathrm{~mm} \mathrm{~V}^{-1}$ obtained by the contact calibration falls within the confidence range of the purge calibration method.

\section{Dynamic calibration}

In the normal operation of the Stirling cryocooler, the gas force is provided by the actuating piston oscillating in the compression cylinder with a phase shift relative to the displacer motion. One might raise the question: could we use the static sensitivity calibrated by the contact or purge method for the measurement of the oscillating motion of the displacer? Or, could we calibrate the sensitivity as the displacer oscillates at its normal operating frequency? From the specification, the LVDT behaves as an electromechanical low-pass filter. The measurement gain of the LVDT is a constant within its bandwidth, which is about one-tenth of the AC excitation frequency. The LVDT that we used is designed to be excited by $2500 \mathrm{~Hz}$, so the useful range of motion frequencies is limited to about $250 \mathrm{~Hz}$. This is adequate for measuring the displacer with $20-40 \mathrm{~Hz}$ motion frequencies.

Therefore, the static sensitivity obtained by the contact or purge calibration must be the same as that by dynamic calibration in the operating range of the cooler. To prove this, the displacer motion, or the stroke, is directly recorded by the optical displacement sensor, after replacing the stainless-steel cap at the bottom of the cold finger with a transparent acrylic cap. The sensitivity is then calculated by dividing the displacer stroke by the corresponding voltage difference read by the I.VDT. Figure 4 shows the measurement sensitivity of the LVDT $28 \mathrm{~mm}$ from the base of the cold finger for various cold-tip temperatures measured with a T-type thermocouple. The average sensitivity over the temperature range of -150 to $0^{\circ} \mathrm{C}$ is $12.63 \mathrm{~mm} \mathrm{~V}^{-1}$, which is quite reliable and very close to the sensitivity of $12.12 \mathrm{~mm} \mathrm{~V}^{-1}$ obtained by the contact calibration.

\section{Temperature compensation}

In the above calibrations, the LVDT voltage output was dependent upon the tip temperature of the cold finger. To investigate the temperature effect on the voltage output of LVDT located, for example, $28 \mathrm{~mm}$ from the base of the cold finger, the Stirling cryocooler operated until the temperature of the cold tip decreased to $-130^{\circ} \mathrm{C}$. Then the operation was stopped and the LVDT output voltage recorded as the temperature rose. At the same time, the optical displacement meter detected no movement of the displacer. It can be assumed that the tip temperature of the cold finger 


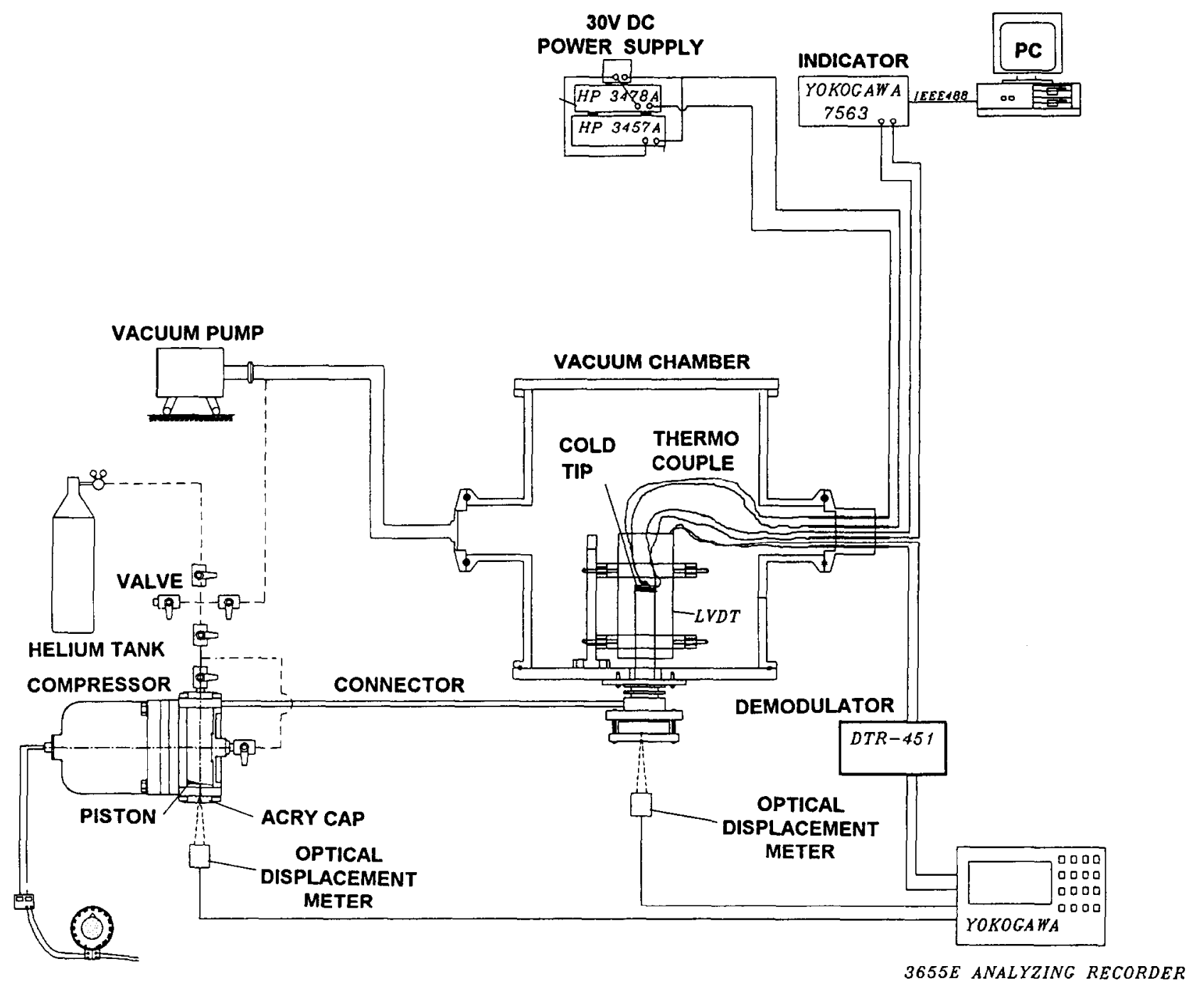

Figure 1 Measurement apparatus

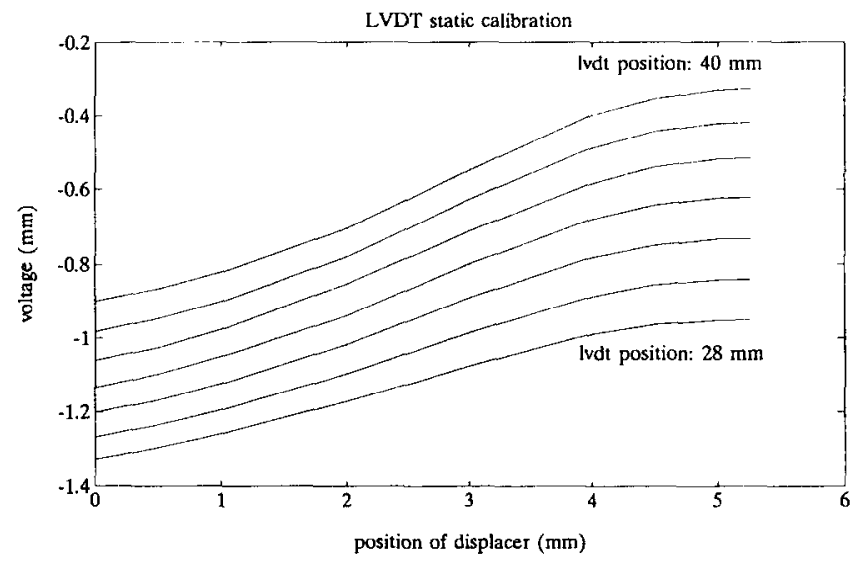

Figure 2 Contact calibration curves

is an explicitly dependent variable on the voltage shift read by the LVDT.

Figure 5 shows that the sensitivity is approximately kept constant over the temperature range $20^{\circ} \mathrm{C}$ to $-120^{\circ} \mathrm{C}$, i.e. the sensitivity is independent of temperature. Therefore, the $K$ value obtained from the purge calibration at room temperature is adopted. The voltage curve versus tip temperature, as shown in Figure 5, was successfully fitted by a linear curve of gradient $\frac{\mathrm{d} V}{\mathrm{~d} T}=-0.0027 \mathrm{~V}^{\circ} \mathrm{C}^{-1}$

with a linear correlation coefficient of -0.9996 , where $V$ is the output voltage of the LVDT and $T$ denotes the coldtip temperature in ${ }^{\circ} \mathrm{C}$. Therefore, Equation (2) is modified as follows:

$x(t)=K\left[V(T, t)-V_{0}-\left(T-T_{\mathrm{amb}}\right) \frac{\mathrm{d} V}{\mathrm{~d} T}\right]$

where $T_{\text {amb }}$ is the ambient temperature at which $V_{0}$ is measured. Figures 6 and 7 present the displacer motion measured by the optical displacement meter and by the LVDT without and with temperature compensation, respectively, at $-130^{\circ} \mathrm{C}$ and a $33 \mathrm{~Hz}$ operating frequency. Notice in Figure 8 the stroke of the displacer motion measured by the LVDT with temperature compensation is $10 \%$ lower than the stroke measurement from the optical displacement meter. This difference occurs due to the sensor nonlinearities, calibration errors, misalignments, etc., and is acceptable from an engineering point of view. 
Non-contact measurement of displacer motion: Y.P. Yang et al.

Table 1 Static calibration of sensitivity

\begin{tabular}{|llll|}
\hline $\begin{array}{l}\text { LVDT position } \\
(\mathrm{mm})\end{array}$ & $\begin{array}{l}\text { Sensitivity } \\
\left(\mathrm{mm} \mathrm{V}^{-1}\right)\end{array}$ & Offset & Correlation coefficient \\
\hline 28 & 12.12 & 17.02 & 0.9947 \\
30 & 11.26 & 14.32 & 0.9938 \\
32 & 10.15 & 12.27 & 0.9934 \\
34 & 9.32 & 10.65 & 0.9936 \\
36 & 8.76 & 9.41 & 0.9943 \\
38 & 8.41 & 8.42 & 0.9936 \\
40 & 8.27 & 7.63 & 0.9940 \\
\hline
\end{tabular}

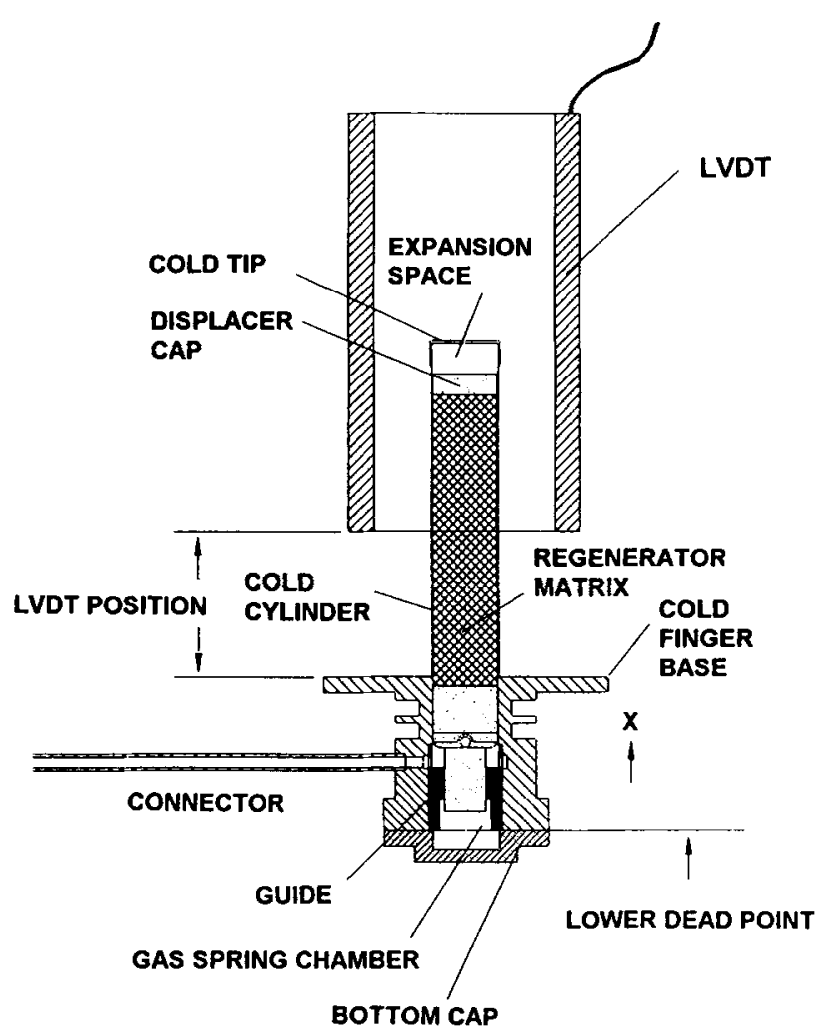

Figure 3 Setup of sensor and cold finger

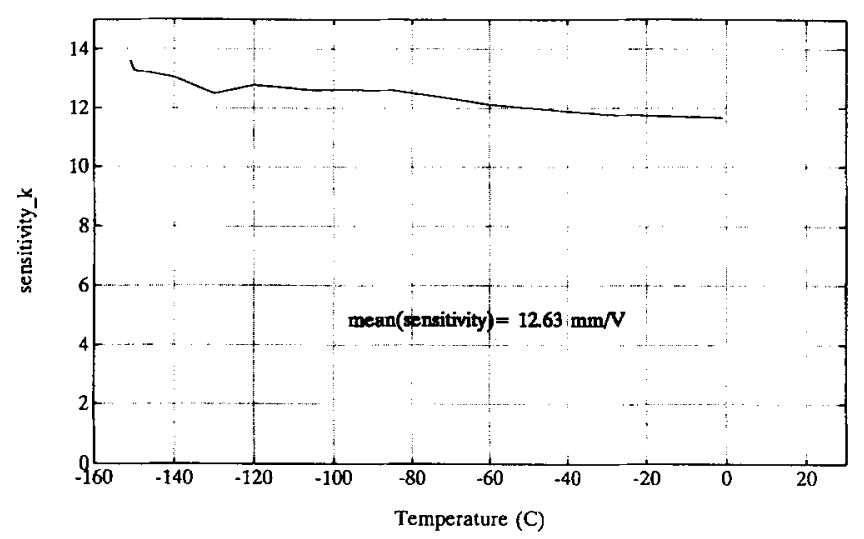

Figure 4 Dynamical calibration curve versus cold-end temperature

\section{Summary and conclusions}

Three optional methods have been presented to calibrate the static sensitivity of measurement of the displacer motion, by which the induced voltage of the LVDT is very low due to the weak magnetic permeability of the regener-

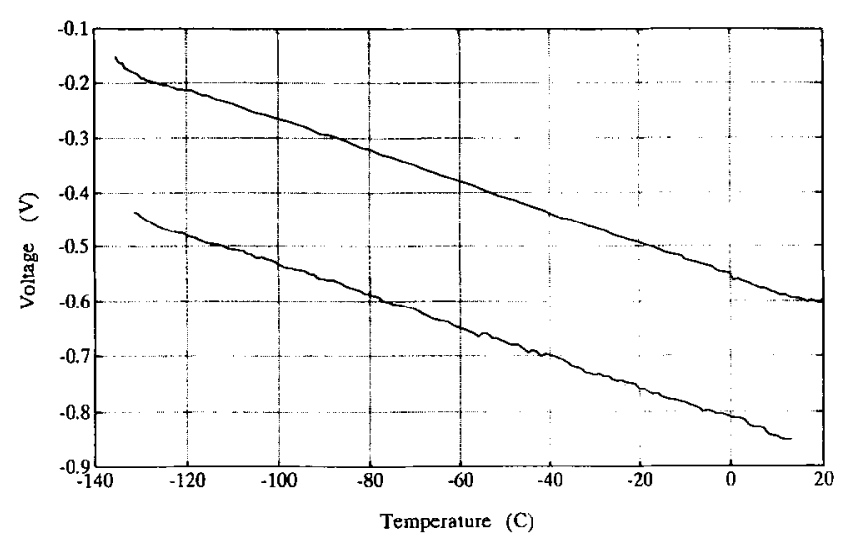

Figure 5 Temperature compensation experimental curve

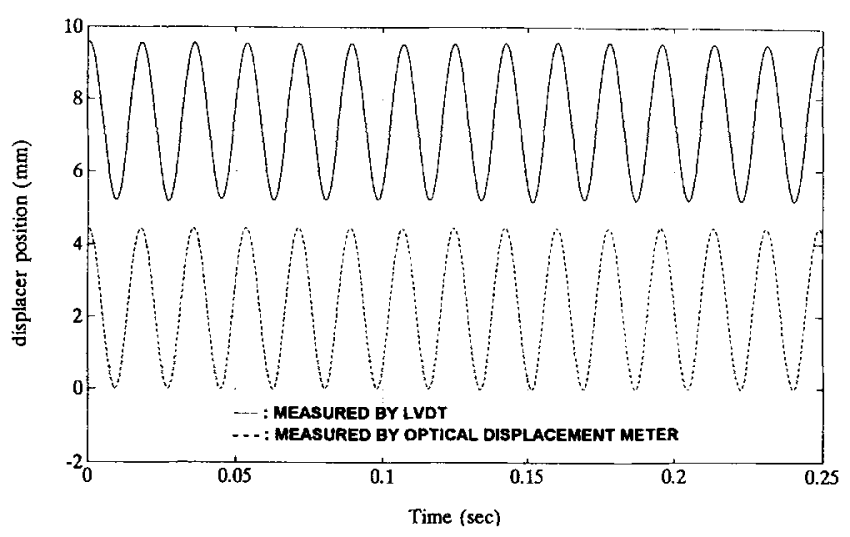

Figure 6 Comparison of outputs from optical displacement meter and LVDT without temperature compensation at $-130^{\circ} \mathrm{C}$

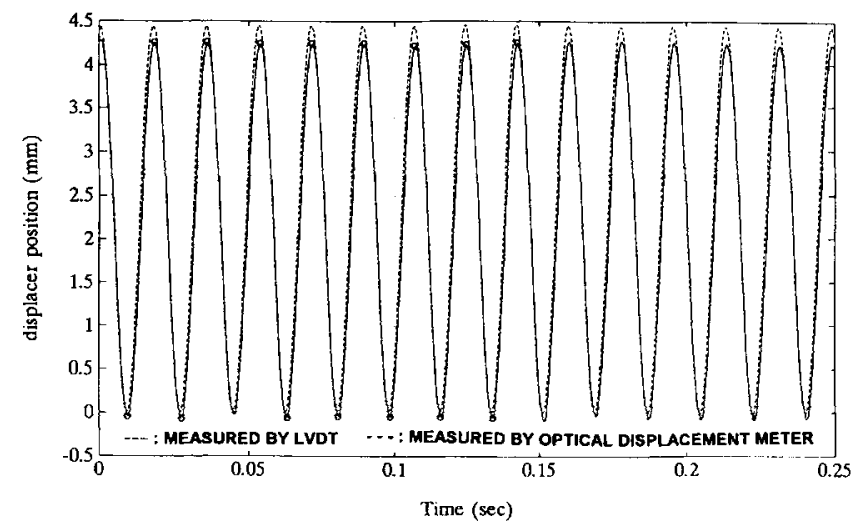

Figure 7 Comparison of outputs from optical displacement meter and LVDT with temperature compensation at $-130^{\circ} \mathrm{C}$ 


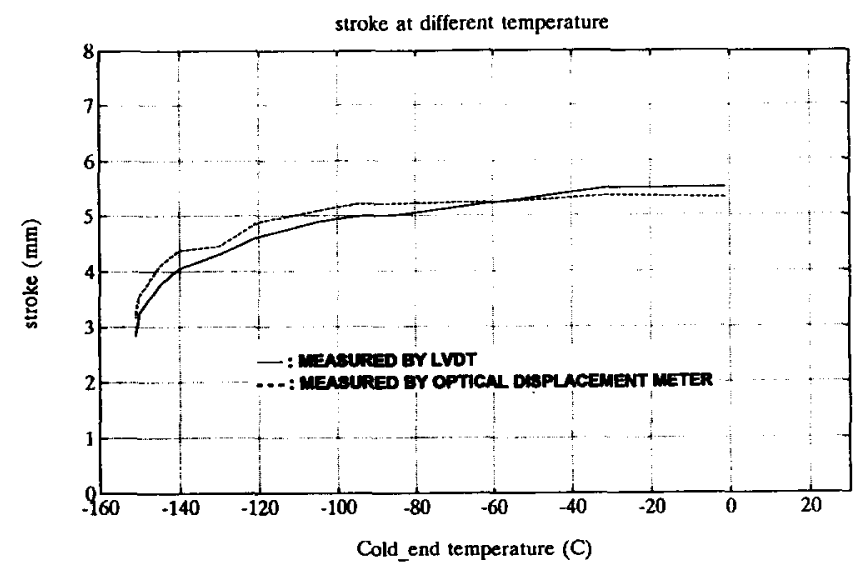

Figure 8 Displacer stroke with temperature compensation

ator matrix and shielding effect of the cold cylinder. This does not mean that the previously proposed relative displacement measurement method does not contribute to the calibration of static sensitivity, but is limited to the regenerator matrix of high magnetic permeability relative to the cold cylinder. The contact calibration method is used when the bottom cap of the cold finger is removed. This is not practical since the cryocooler must be disassembled. The purge calibration can be performed during the working gas charging process. The dynamic calibration helps us prove that the sensitivity calibrated by static calibrations can be used in the measurement of the dynamic motion of the displacer. The values of static sensitivity obtained from all three methods are very close for the same location of the LVDT, although the sensitivity is a nonlinear function of the position of the LVDT. These calibration methods are very useful and are usually conducted before the prototype or product assembly of the split-Stirling cryocooler. In addition, the true displacer motion must be calculated with temperature compensation, although the displacer stroke is irrelevant to such compensation. Based on this research, anyone working on the same system, either in the laboratory or in product quality control, can use the same measurement technique with a less expensive LVDT without further proof from any other costly instruments.

\section{Acknowledgements}

The authors greatly acknowledge the support of the National Science Council under Contract No. NSC82-0401D002-033 in Taiwan, Republic of China.

\section{References}

1 De Silva, C.W. Control Sensors and Actuators Prentice Hall, Englewood Cliffs, NJ (1989)

2 Xiang, Y., Li, Q. and Guo, F. Identification of the negative feedback relationship in split cycle free piston Stirling cryocooler system Cryogenics (September Supplement 1990) 30 216-220

3 Zhang, T., Tan, L., Li, Z. and Zhang, D. Experimental investigation on the dynamic pressure distribution in a split piston Stirling cryocooler system Cryogenics (September Supplement 1990) 30 221-225

4 Stolfi, F. and de Jonge, A. K. Stirling cryogenerators with linear drive Philips Technical Review (1985) $421-10$

5 Jones, B. G. and Scull, S. R. Development and preflight qualification testing of a range of cryogenic coolers for applications from $20 \mathrm{~K}$ to $80 \mathrm{~K}$ Cryogenics (1992) 32 850-858

6 Yang, Y. P., Chien, H. T. and Chen, J. M. Non-contact measurement of displacer motion of a miniature split-Stirling cryocooler Measurement (1995) 14 199-208

7 Nohara, K., Kato, T. and Ejima, A. Cryogenic properties of vanadium and nitrogen bearing AISI-316LN type stainless steel for superconduction magnet New Developments in Stainless Steel Technologies, American Society for Metals Conf Proc American Society for Metals, Metals Park, OH, USA (1985) 243-251

8 Walker, G. Miniature Refrigerators for Cryogenic Sensors and Cold Electronics Clarendon Press, Oxford, UK (1989)

9 Hashimoto, T., Ogawa, M. and Li, R. Recent advance in magnetic regenerator material Cryogenics (September Supplement 1990) 30 192-198

10 Doebelin, E. O. Measurement Systems - Application and Design 4th Edn, McGraw-Hill Publishing Company, New York, USA (1990)

11 Cheng, D. K. Field and Wave Electromagnetics Addison-Wesley Publishing Company, Reading, MA, USA (1989)

\section{Appendix}

\section{Specifications of LVDT}

$\begin{array}{ll}\text { Model } & 253 \mathrm{XS}-\mathrm{A}, \mathrm{S} / \mathrm{N} \text { 1454, SCHAEVITZ } \\ \text { Range } & \pm 6 \mathrm{~mm} \\ \text { Body length } & 79.5 \mathrm{~mm} \\ \text { Sensitivity } & 32 \mathrm{mV} \mathrm{mm}^{-1} \text { (for standard iron core) } \\ \text { Excitation } & 3 \mathrm{VAC}, 2500 \mathrm{~Hz} \\ \text { Primary coil reactance } & 170 \Omega \\ \text { Secondary coil reactance } & 2850 \Omega \\ \text { Linearity } & <0.5 \% \\ \text { Phase } & 3^{\circ}\end{array}$

\section{Specifications of optical displacement sensor}

$\begin{array}{ll}\text { Model } & \text { PA 1830, Keyence Corp. } \\ \text { Reference distance } & 40 \mathrm{~mm} \pm 1 \mathrm{~mm} \\ \text { Measurement range } & \pm 5 \mathrm{~mm} \\ \text { Resolution } & 10 \mu \mathrm{m} \\ \text { Accuracy } & \pm 20 \mu \mathrm{m} \pm 1 \% \text { of measured value }\end{array}$

